\title{
Australian clinical trial activity and burden of disease: an analysis of registered trials in National Health Priority Areas
}

\section{Australian \\ clinical trial \\ activity for \\ obesity and \\ dementia \\ interventions \\ falls below \\ their relative \\ disease burden}

MPharm, BS

Sarah JLord

MBBS, MS, FRACGP

Kylie E Hunter

BA(Human Movement) (Hons), BA(Indonesian)

R John Simes MBBS(Hons), MD, FRACP

Thuyen Vu BSC

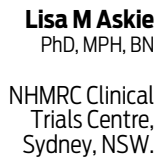

Sally.Lord@ ctc.usyd.edu.au

doi: 10.5694/mjal4.00598
$\mathrm{T}$ o improve Australia's health, clinical research programs should devote substantial activity to advancing practice in areas of high clinical need. Clinical trials are designed to provide high-quality evidence of the effectiveness of new interventions to establish best clinical practice. However, few studies have examined the extent to which Australian clinical trials address priority areas of clinical need.

The Australian Institute of Health and Welfare (AIHW) National Health Priority Areas (NHPAs) were introduced to encourage appropriate targeting of health services and clinical research to improve health. Currently, there are nine NHPAs: cancer control, cardiovascular health, mental health, injury prevention and control, diabetes mellitus, obesity, arthritis and musculoskeletal conditions, dementia and asthma. These NHPAs account for approximately three-quarters of the total estimated burden of disease in Australia (1915600 of 2632800 disability-adjusted life-years [DALYs]). ${ }^{1}$

Previous studies have reported a disparity between the level of National Health and Medical Research Council (NHMRC) grant funding for studies investigating NHPA conditions relative to their disease burden..$^{2,3}$ The founding of clinical trial registries, including the Australian New Zealand Clinical Trial Registry (ANZCTR) in 2005, provides the first opportunity to examine how well clinical trial activity in Australia is targeted to NHPAs.

\section{Methods}

We conducted a retrospective analysis using ANZCTR and ClinicalTrials. gov (CT.gov) data to report on Australian trial activity and characteristics for NHPAs; and to compare the level of trial activity to the relative burden of disease for each NHPA.

\section{Abstract}

Objective: To assess whether Australian clinical trials activity in National Health Priority Areas (NHPAs) reflects the relative disease burden.

Design and setting: Analysis of trials registered on the Australian New Zealand Clinical Trials Registry (ANZCTR) or ClinicalTrials.gov from January 2008 to December 2012 that planned recruitment in Australia and investigated interventions for NHPA conditions (cancer control, cardiovascular health, mental health, obesity, injury prevention/control, diabetes mellitus, arthritis and musculoskeletal conditions, dementia and asthma). Australian estimates of disability-adjusted life-years (DALYs) were used to quantify the burden of disease for each NHPA.

Main outcome measures: For each NHPA, the total number of registered trials, planned recruitment, and the predicted numbers based on disabilityadjusted life-years expressed as a proportion of the total burden of disease in Australia (\%DALY).

Results: 5143 trials with Australian sites were registered in the 5-year study period with total planned recruitment of 2404609 participants. Of these, 3032 trials (59\%) with planned recruitment of 1532064 participants (64\%) investigated NHPA conditions. Trial numbers and planned recruitment were highest for cancer, cardiovascular and mental health reflecting their higher disease burden. In contrast, planned recruitment into obesity and dementia trials was $\leqslant 50 \%$ of that predicted from total trial activity based on their relative disease burden. The number of registered trials for these conditions was also lower than predicted. Overall, of 3032 NHPA trials, 2335 (77\%) used randomisation and 1520 (50\%) planned to recruit $>100$ participants.

Conclusions: Australian clinical trial activity for obesity and dementia interventions is lower that would be expected based on their relative disease burden. Trial registries provide a valuable public database to identify and monitor gaps in research activity.

Ethics approval was not required for this analysis of publicly available trial data.

\section{Data sources}

Trial registration is voluntary in Australia. ${ }^{4}$

The ANZCTR is an online public registry of clinical trials maintained by the NHMRC Clinical Trials Centre, the University of Sydney. It collects information about trial interventions, investigated health conditions, planned recruitment, outcomes, funding and sponsorship using the World Health Organization-defined 20-item minimum dataset. ${ }^{5}$ Health conditions are coded using the United Kingdom Clinical Research Collaboration Health Research Classification System (http://www. hrcsonline.net). Additional data are collected about trial design, including randomisation and blinding. The ANZCTR 2011 Data Quality and Completeness Audit reported that, on average, at least 93 of 94 data fields for 148 trials were complete. ${ }^{6}$

CT.gov is an online public registry of clinical trials maintained by the United States National Library of Medicine (https://clinicaltrials.gov). It records similar data items to the ANZCTR.

\section{Trial sample and characteristics}

The trial sample included all trials of health-related interventions registered on the ANZCTR or CT.gov between 1 January 2008 and 31 December 2012 that included Australia as a country of recruitment. 
To avoid entering duplicate trial data, trials that listed a CT.gov or ANZCTR registration number as a secondary identifier were only included in the ANZCTR trial list.

Condition categories and codes were used to classify individual trials as addressing one or more NHPA conditions, or other, non-NHPA conditions. For each trial, we extracted information for: purpose of intervention (treatment, prevention, diagnosis, education/counselling/ training, other/missing); allocation of intervention (randomised, non-randomised); trial phase (I-IV, not applicable, missing), blinding (blinded, open, other/missing), planned recruitment (reported as target sample size, and classified as $<100,100-1000$, > 1000 participants); participant age range $(<18$ years, $18-69$ years, $\geqslant 70$ years); and countries of recruitment (Australia only, Australia and overseas).

\section{Analysis}

To measure trial activity, we recorded the total number and planned recruitment of registered trials investigating NHPA conditions. To assess whether trial activity reflected the burden of disease for each NHPA, we compared the relative trial activity targeted to each NHPA, measured as a proportion of the total trial activity, with the "expected" distribution of trial activity estimated from the relative burden of disease for that NHPA. Burden of disease was estimated from published estimates of DALYs for each NHPA expressed as a percentage of the total burden of disease and injury in Australia (\%DALY). ${ }^{1}$

To describe disparities in relative trial activity by NHPA, we identified NHPAs where the observed trial activity was less than $50 \%$ or more than $200 \%$ of expected values. The $\chi^{2}$ goodness-of-fit test was also used to test for statistically significant differences between observed and expected trial activity for each NHPA. For these analyses, a twosided $P<0.006$ was regarded as statistically significant using the Bonferroni adjustment for multiple comparisons (nine comparisons).

For assessment of trial recruitment across NHPA, we also conducted a sensitivity analysis to examine trial recruitment to NHPA from Australian sites, where Australian recruitment was estimated from the planned recruitment from all ANZCTR trials plus $10 \%$ of the planned recruitment from CT.gov trials that included at least one Australian site. The figure of
$10 \%$ was estimated from a randomly selected sample of $100 \mathrm{CT}$.gov registered trials that included at least one Australian site and represents the number of Australian sites as a proportion of all sites for each trial.

We also calculated the frequency distribution of trial characteristics for each NHPA. SAS, version 9.3 (SAS Institute) was used for data analyses.

\section{Results}

There were 5143 intervention trials registered during 2008-2012 that planned to recruit in Australia (ANZCTR, 3379; CT.gov, 1764). Of these, 3032 (59\%) related to NHPA conditions (ANZCTR, 1908; CT.gov, 1124). Total planned recruitment for the trial sample was 2404609 participants, including 1532064 (64\%) for NHPA trials (ANZCTR, 670832; CT.gov, 861 232).

\section{Trial activity in NHPA}

The three disease areas that contribute the largest \%DALY - cancer, cardiovascular diseases and mental disorders - also attracted the largest number of trial registrations and the largest planned recruitment (Box 1; Box 2).

\section{Number of registered Australian intervention trials and total planned recruitment in National Health Priority Areas, as a percentage of total trial activity, and comparison to the expected number based on \%DALY, Australian New Zealand Clinical Trials Registry and ClinicalTrials.gov, 2008-2012}

\begin{tabular}{|c|c|c|c|c|c|c|c|c|c|c|c|c|}
\hline \multirow[b]{2}{*}{ National Health Priority Area } & \multicolumn{2}{|c|}{ DALY } & \multicolumn{5}{|c|}{ Trials } & \multicolumn{5}{|c|}{ Planned recruitment } \\
\hline & Rank & $\%$ & Rank & $\begin{array}{l}\text { Observed } \\
\text { no. }(\%)\end{array}$ & $\begin{array}{c}\text { Expected } \\
\text { no. }\end{array}$ & $\begin{array}{l}\text { Observed/ } \\
\text { expected } \%\end{array}$ & $P *$ & Rank & $\begin{array}{l}\text { Observed } \\
\text { no. (\%) }\end{array}$ & $\begin{array}{c}\text { Expected } \\
\text { no. }\end{array}$ & $\begin{array}{l}\text { Observed/ } \\
\text { expected \% }\end{array}$ & $P^{*}$ \\
\hline Cancer control & 1 & $19.0 \%$ & 1 & $871(16.9 \%)$ & 977 & $89 \%$ & 0.007 & 2 & $\begin{array}{l}427188 \\
(17.8 \%)\end{array}$ & 456876 & $94 \%$ & $<0.001$ \\
\hline Cardiovascular health & 2 & $18.0 \%$ & 3 & $646(12.6 \%)$ & 926 & $70 \%$ & $<0.001$ & 1 & $\begin{array}{l}577178 \\
(24.0 \%)\end{array}$ & 432830 & $133 \%$ & $<0.001$ \\
\hline Mental health & 3 & $13.3 \%$ & 2 & $693(13.5 \%)$ & 684 & $101 \%$ & 0.82 & 3 & $\begin{array}{l}196826 \\
(8.2 \%)\end{array}$ & 319813 & $62 \%$ & $<0.001$ \\
\hline Obesity & 4 & $7.5 \%$ & 6 & $195(3.8 \%)$ & 386 & $51 \%$ & $<0.001$ & 7 & $33948(1.4 \%)$ & 180346 & $19 \%$ & $<0.001$ \\
\hline Injury prevention and control & 5 & $7.0 \%$ & 7 & $137(2.7 \%)$ & 360 & $38 \%$ & $<0.001$ & 5 & $125256(5.2 \%)$ & 168323 & $74 \%$ & $<0.001$ \\
\hline Diabetes mellitus & 6 & $5.5 \%$ & 5 & $282(5.5 \%)$ & 283 & $100 \%$ & 1.00 & 4 & $185929(7.7 \%)$ & 132253 & $141 \%$ & $<0.001$ \\
\hline $\begin{array}{l}\text { Arthritis and musculoskeletal } \\
\text { conditions }\end{array}$ & 7 & $4.0 \%$ & 4 & $410(8.0 \%)$ & 206 & $199 \%$ & $<0.001$ & 6 & 109107 (4.5\%) & 96184 & $113 \%$ & $<0.001$ \\
\hline Dementia & 8 & $3.6 \%$ & 9 & 65 (1.3\%) & 185 & $35 \%$ & $<0.001$ & 9 & $24248(1.0 \%)$ & 86566 & $28 \%$ & $<0.001$ \\
\hline Asthma & 9 & $2.4 \%$ & 8 & $68(1.3 \%)$ & 123 & $55 \%$ & $<0.001$ & 8 & $29468(1.2 \%)$ & 57711 & $51 \%$ & $<0.001$ \\
\hline
\end{tabular}

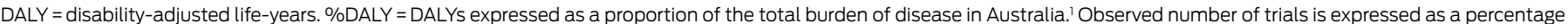

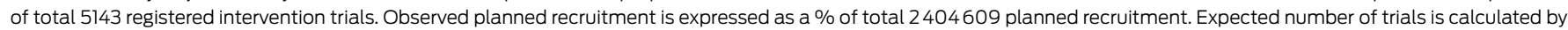
applying \%DALY to total 5143 registered intervention trials. Expected planned recruitment is calculated by applying \%DALY to total 2404609 planned recruitment.

$* \chi^{2}$ goodness-of-fit test for comparison of observed versus expected values. 
The proportions of registered trials that investigated dementia or injury interventions were less than half those expected from their \%DALYs (65/185 [35\%] and 137/360 [38\%], respectively; Box 1). The proportions of obesity and asthma trials were also lower than expected (195/386 [51\%] and 68/123 [55\%], respectively). In contrast, the proportion of registered arthritis and musculoskeletal diseases trials was about twice as high as expected on the basis of the \%DALY (Box 1).

The proportions of planned recruitment to trials investigating obesity and dementia were also substantially lower than expected from their \%DALYs (33948/180346 [19\%] and 24248/86566 [28\%], respectively), and was also low for asthma (29468/57711 [51\%]) (Box 1).

When this analysis was repeated using estimated recruitment from Australian sites only, a similar pattern was observed, with the exception of recruitment to diabetes trials. For diabetes trials, total trial planned recruitment was relatively high (185929/132253 [141\%]) compared with Australian sites (44201/66607 [66\%]).

\section{Trial characteristics}

Overall, 2335 of 3032 (77\%) NHPA trials used a randomised design and 1509 (50\%) planned recruitment of $\leqslant 100$ participants (Box 3 ). Of the 2931 NHPA trials that reported information about blinding, 1504 (51\%) reported using it (Box 3).

About three-quarters of NHPA intervention trials investigated treatments (2321 [76\%]) and 397 (13\%) investigated prevention interventions (Box 3). The ratio of treatment to prevention trials ranged from less than $2: 1$ for obesity trials to $14: 1$ for cancer trials.

Most NHPA trials excluded children, whereas 2252 (75\%) specified a maximum participant age of $\geqslant 70$ years, or did not specify a maximum age (Box 3). International recruitment sites were reported in 1081 (36\%) of NHPA trials (169 ANZCTR trials, 912 CT.gov trials) and varied by condition (Box 3).

\section{Discussion}

This study provides the first overview of clinical trial activity in Australia. We found that more than half of Australian registered intervention trials and planned trial recruitment are targeted to NHPA conditions.

Trial activity for cancer, cardiovascular diseases and mental disorders was high relative to other NHPA conditions, consistent with their position as the three major contributors to disability and premature death in Australia. In contrast, trial activity for obesity and dementia interventions was substantially less than the level expected from their contribution to the total DALY.

To interpret these results, the number of trials can be considered to provide a proxy measure for the number of active research questions being investigated to identify more effective interventions in each area. Planned trial recruitment provides a measure of the number of patients actively participating in research to determine best practice in each area.

These findings suggest there is a need to further examine research activity for obesity, dementia and asthma to determine if and how clinical trials research in these areas should be increased. However, this study does not allow us to define the optimum level of trial activity for each condition. Clearly, not all important research questions for NHPAs are amenable to investigation through clinical trials. For conditions where trial activity is already high relative to other disease areas, further increases may still represent good value for money by improving health care. For example, if promising new interventions are available; or practice variations or controversies exist with gaps in evidence to guide best practice. Conversely, for some conditions where trial activity is currently low, research priorities may warrant other study designs, such as those used in translational research or behavioural science, to develop new interventions.

This study also provides the first opportunity to assess the extent to which Australian trials are designed
2 Relationship between trial characteristics and \%DALY for each NHPA, Australian New Zealand Clinical Trials Registry and ClinicalTrials.gov, 2008-2012
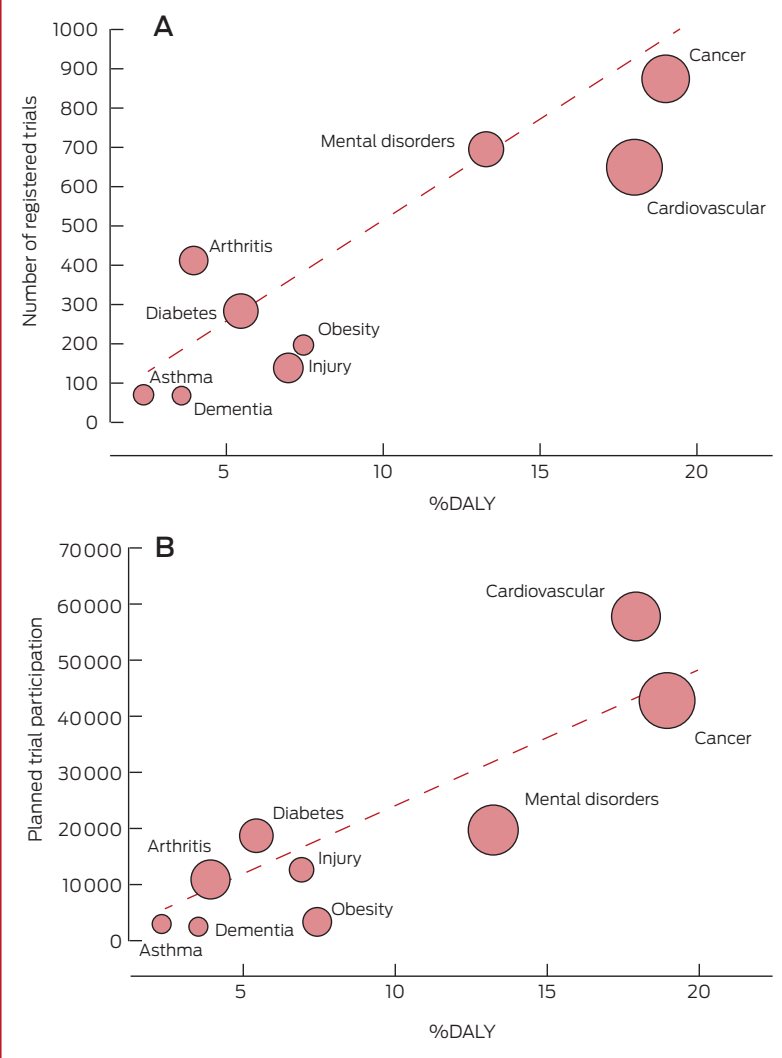

The diagonal line represents the line of equality where \%DALY is equal to trial number as a percentage of total registered trials $(\boldsymbol{A})$ or planned trial participation as \% of total planned trial participation (B). Dots below the line show NHPAs where the variable falls below the \%DALY. The size of dots corresponds to the size of planned trial participation $(\boldsymbol{A})$ or number of trials $(B)$ for the NHPA.

$\% D A L Y=$ disability-adjusted life-years expressed as a proportion of the total burden of disease in Australia.' NHPA = National Health Priority Area.

to provide robust, high-quality evidence for guiding practice. The use of randomisation and blinding provides a measure of trial quality; trial size provides an indicator of study power. Trials enrolling more than 100 participants are generally required to assess clinically meaningful health outcomes and to weigh up the benefits and harms of the new strategy, whereas smaller trials are generally designed to assess surrogate outcomes. About three-quarters of Australian trials used a randomised design; however, only around half reported blinding, or planned recruitment of more than 100 participants. These findings are slightly more favourable than those of a recent analysis of 79413 intervention trials 
3 Australian intervention trial characteristics, overall and by National Health Priority Area (NHPA),* Australian New Zealand Clinical Trials Registry and ClinicalTrials.gov, 2008-2012

\begin{tabular}{|c|c|c|c|c|c|c|c|c|c|c|c|}
\hline Characteristic & All trials & $\begin{array}{l}\text { NHPA } \\
\text { trials }\end{array}$ & Cancer & $\begin{array}{l}\text { Cardio- } \\
\text { vascular }\end{array}$ & $\begin{array}{l}\text { Mental } \\
\text { health }\end{array}$ & Obesity & Injury & Diabetes & $\begin{array}{c}\text { Arthritis/ } \\
\text { musculoskeletal }\end{array}$ & Dementia & Asthma \\
\hline Total & 5143 & 3032 & 871 & 646 & 693 & 195 & 137 & 282 & 410 & 65 & 68 \\
\hline \multicolumn{12}{|l|}{ Randomisation } \\
\hline Yes & 3990 (78\%) & $2335(77 \%)$ & $564(65 \%)$ & $494(77 \%)$ & $579(84 \%)$ & $163(84 \%)$ & $125(91 \%)$ & $253(90 \%)$ & $321(78 \%)$ & $53(82 \%)$ & $59(87 \%)$ \\
\hline No & 1137 (22\%) & $691(23 \%)$ & 304 (35\%) & $150(23 \%)$ & $113(16 \%)$ & $31(16 \%)$ & $12(9 \%)$ & $28(10 \%)$ & $89(22 \%)$ & $12(18 \%)$ & $9(13 \%)$ \\
\hline Missing & 16 & 6 & 3 & 2 & 1 & 1 & & 1 & & & \\
\hline \multicolumn{12}{|l|}{ Intervention type } \\
\hline Treatment & $3834(75 \%)$ & $2321(76 \%)$ & $732(84 \%)$ & $444(69 \%)$ & 494 (71\%) & $108(55 \%)$ & $103(75 \%)$ & $210(75 \%)$ & $357(87 \%)$ & $50(77 \%)$ & $46(68 \%)$ \\
\hline Prevention & $781(15 \%)$ & $397(13 \%)$ & $52(6 \%)$ & $131(20 \%)$ & $98(14 \%)$ & $67(34 \%)$ & $25(18 \%)$ & $46(16 \%)$ & $34(8 \%)$ & $5(8 \%)$ & $10(15 \%)$ \\
\hline Diagnosis & $152(3 \%)$ & $78(3 \%)$ & $29(3 \%)$ & $26(4 \%)$ & $11(2 \%)$ & $3(2 \%)$ & $2(2 \%)$ & $8(3 \%)$ & $4(1 \%)$ & $4(6 \%)$ & 0 \\
\hline $\begin{array}{l}\text { Educational/ } \\
\text { counselling/training }\end{array}$ & $263(5 \%)$ & $171(6 \%)$ & $39(5 \%)$ & $26(4 \%)$ & $73(11 \%)$ & $10(5 \%)$ & $4(3 \%)$ & $15(5 \%)$ & $9(2 \%)$ & $5(8 \%)$ & $7(10 \%)$ \\
\hline Other/missing & $113(2 \%)$ & $65(2 \%)$ & $19(2 \%)$ & $19(3 \%)$ & $17(2 \%)$ & $7(4 \%)$ & $3(2 \%)$ & $3(1 \%)$ & $6(2 \%)$ & $1(2 \%)$ & $5(7 \%)$ \\
\hline \multicolumn{12}{|l|}{ Age group (years) } \\
\hline Minimum age $<18^{\dagger}$ & $987(19 \%)$ & $490(16 \%)$ & $122(14 \%)$ & $60(9 \%)$ & $156(23 \%)$ & $29(15 \%)$ & $42(31 \%)$ & $28(10 \%)$ & $57(14 \%)$ & $7(11 \%)$ & $26(38 \%)$ \\
\hline Missing & 5 & 2 & 1 & & & & & & & 1 & \\
\hline Maximum age $\geqslant 70^{\dagger}$ & 3652 (71\%) & $2252(75 \%)$ & 774 (89\%) & $558(87 \%)$ & $397(57 \%)$ & $69(36 \%)$ & $98(72 \%)$ & 199 (71\%) & $316(77 \%)$ & $59(94 \%)$ & 41 (60\%) \\
\hline Missing & 18 & 10 & 2 & 2 & & 1 & & & 2 & 2 & \\
\hline \multicolumn{12}{|l|}{ Blinding } \\
\hline Blinded & 2639 (53\%) & $1504(51 \%)$ & $270(31 \%)$ & $347(55 \%)$ & 405 (61\%) & $93(51 \%)$ & $89(67 \%)$ & $141(52 \%)$ & 249 (64\%) & $47(72 \%)$ & $48(72 \%)$ \\
\hline Open & $2322(47 \%)$ & $1427(49 \%)$ & $589(69 \%)$ & $281(45 \%)$ & $260(39 \%)$ & 91 (49\%) & $43(33 \%)$ & $129(48 \%)$ & $139(36 \%)$ & $18(28 \%)$ & $19(28 \%)$ \\
\hline Missing & 182 & 101 & 12 & 18 & 28 & 11 & 5 & 12 & 22 & 0 & 1 \\
\hline \multicolumn{12}{|l|}{$\begin{array}{l}\text { Planned } \\
\text { recruitment }\end{array}$} \\
\hline $1-100$ & $2689(52 \%)$ & 1509 (50\%) & 361 (41\%) & $325(50 \%)$ & $361(52 \%)$ & $132(68 \%)$ & $66(48 \%)$ & $133(47 \%)$ & $228(56 \%)$ & $22(35 \%)$ & $33(49 \%)$ \\
\hline 101-1000 & 2066 (40\%) & $1274(42 \%)$ & $427(49 \%)$ & $244(38 \%)$ & $300(43 \%)$ & $58(30 \%)$ & $61(45 \%)$ & $119(42 \%)$ & $161(39 \%)$ & $35(55 \%)$ & $31(46 \%)$ \\
\hline$>1000$ & $383(7 \%)$ & $246(8 \%)$ & $83(10 \%)$ & $77(12 \%)$ & $30(4 \%)$ & $5(2 \%)$ & $10(7 \%)$ & $30(11 \%)$ & $21(5 \%)$ & $6(10 \%)$ & $3(5 \%)$ \\
\hline Missing & 5 & 3 & 1 & & 2 & & & & & 2 & 1 \\
\hline \multicolumn{12}{|c|}{ Country of recruitment } \\
\hline Australia only & $3521(68 \%)$ & $1951(64 \%)$ & $349(40 \%)$ & $401(62 \%)$ & $578(83 \%)$ & $184(94 \%)$ & $113(82 \%)$ & $192(68 \%)$ & $286(70 \%)$ & $37(57 \%)$ & $47(69 \%)$ \\
\hline $\begin{array}{l}\text { Australia and } \\
\text { overseas }\end{array}$ & $1622(32 \%)$ & $1081(36 \%)$ & $522(60 \%)$ & $245(38 \%)$ & $115(17 \%)$ & $11(6 \%)$ & $24(18 \%)$ & $90(32 \%)$ & $124(30 \%)$ & $28(43 \%)$ & $21(31 \%)$ \\
\hline
\end{tabular}

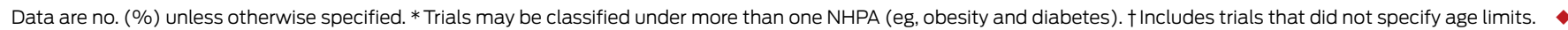

registered on CT.gov between 2000 and 2010, which reported that $70 \%$ used a randomised design, $44 \%$ used a blinded design and 38\% enrolled 100 or more participants. ${ }^{7}$

One commonly raised concern about clinical trials research is the applicability of trial data to routine clinical practice populations and settings. Our finding that more than twothirds of trials in NHPA areas did not exclude participants aged 70 years or older is encouraging.

The main strength of our study is that it provides a unique, timely overview of Australian clinical trials to inform current debate on the achievements, limitations and future directions for clinical trials research in Australia. Clinical researchers can use the same methods to further explore gaps for conditions within specific disease areas, as has been performed for cancer trials. ${ }^{8}$

There are two main limitations to our study that could affect our estimates of trial activity in different directions. First, we relied on trial registrations to estimate trial activity. As trial registration is not compulsory in Australia, we may have underestimated trial activity. Additionally, we only included international trials registered on the ANZCTR or CT.gov. A search using the WHO International Clinical Trials Registry Platform Search Portal (http://www.who.int/ictrp/search/ en) showed that 11096 of 11412 (97\%) trials with Australian sites are registered on these two registries. The total number of registered trials may therefore be $3 \%$ higher than our study estimate.

Second, our estimates of trial participation may overestimate the number of Australians participating in 
clinical trials, because 1622 of 5143 trials (32\%) included sites outside Australia. Nevertheless, by including Australian sites, these trial recruitment figures capture participation in trials that can be expected to provide evidence relevant to Australian practice.

Despite these limitations, we believe our findings are valuable in informing initiatives to increase clinical trial activity. ${ }^{9,10}$ It is well documented that trial research is often not available to guide many routine clinical decisions about selecting interventions. ${ }^{11}$ To guide practice, large trials with adequate long-term follow-up are needed to identify small incremental improvements in health outcomes and/or adverse events. Our findings on trial size suggest that further efforts are needed to promote and support the conduct of large trials, or support the conduct of small high-quality trials that can later contribute data to meta-analyses.

Overall, we demonstrate the feasibility and value of using publicly available trial registry data to examine the profile of trials research for particular conditions and identify gaps in trial activity to inform trial initiatives. The ANZCTR provides a valuable resource for researchers to ensure new studies build on, or contribute to, existing trials.

Acknowledgements: The ANZCTR is funded by an NHMRC enabling grant, Therapeutic Innovation Australia, and the Health Research Council of New Zealand.

Competing interests: No relevant disclosures.

Received 22 Aug 2014, accepted 18 Nov 2014.

References are available online at www.mja.com.au. 
1 Australian Institute of Health and Welfare, Begg S, Vos T, Barker B, et al. The burden of disease and injury in Australia 2003. Canberra: AlHW, 2007. (AlHW Cat. No. PHE 82.) http:// www.aihw.gov.au/publicationdetail/?id=6442467990 (accessed Apr 2012).

2 Aoun S, Pennebaker D, Pascal R. To what extent is health and medical research funding associated with the burden of disease in Australia? Aust NZ J Public Health 2004; 28: 80-86.

3 Mitchell RJ, McClure RJ, Olivier J, Watson WL. Rational allocation of Australia's research dollars: does the distribution of NHMRC funding by National Health Priority Area reflect actual disease burden? Med J Aust 2009; 191: 648-652.

4 National Health and Medical Research Council, Australian Research Council, Australian Vice-Chancellors' Committee. National statement on ethical conduct in human research.
Canberra: Australian Government, 2007. www.nhmrc.gov.au/files_ nhmrc/publications/attachments/e72. pdf (accessed Mar 2013).

5 Australian New Zealand Clinical Trial Registry. Data item definition/ explanation. http://www.anzctr. org.au/docs/ANZCTR\%20Data\%20 field\%20explanation.pdf (accessed Apr 2012).

6 Tai F, Ooi W, Hunter K, et al. Data completeness and quality: the results of the Australian New Zealand Clinical Trial Registry 2011 data audits. Cochrane Database Syst Rev 2012; Suppl 1: 13. http://www.cochranelibrary.com/ dotAsset/d5356a06-cddf-42c5-bf24af7a6edbff99.pdf (accessed Jun 2015).

7 Califf RM, Zarin DA, Kramer JM, et al. Characteristics of clinical trials registered in ClinicalTrials.gov, 20072010. JAMA 2012; 307: 1838-1847.

8 Dear RF, Barratt AL, McGeechan K, et al. Landscape of cancer clinical trials in
Australia: using trial registries to guide future research. Med J Aust 2011; 194: 387-391.

9 Clinical Trials Action Group. Clinically competitive: boosting the business of clinical trials in Australia. Clinical Trials Action Group Report. Canberra: Commonwealth of Australia, 2011. http://www.industry.gov.au/ industry/IndustrySectors/Pharma ceuticalsandHealthTechnologies/ ClinicalTrialsActionGroup/Documents/ Clinical_Trials_Action_Group_Report. pdf (accessed Apr 2014).

10 Winship IM, McNeil J, Simes RJ. A funding model for public-good clinical trials. Med J Aust 2013; 199: 90-91.

11 Giannakakis IA, Haidich AB, Contopoulos-loannidis DG, et al. Citation of randomized evidence in support of guidelines of therapeutic and preventive interventions. J Clin Epidemiol 2002; 55: 545-555. 